\title{
Dos NUEVOS REgISTROS DE MUSGOS (BRYOPHYTA) PARA LA Argentina
}

\author{
TWo NEW RECORDS OF MOSSES (BRYOPHYTA) FOR ARgENTINA
}

\author{
Agustina Celeste Cottet $^{1 *}$ (D) y María Inés Messuti ${ }^{1}$
}

\begin{abstract}
1. Instituto de Investigaciones en Biodiversidad y Medioambiente (INIBIOMA); Consejo Nacional de Investigaciones Científicas y Técnicas (CONICET) - Universidad Nacional del Comahue (UNComahue), Quintral 1250, San Carlos de Bariloche, Río Negro - CP 8400 , Argentina.
\end{abstract}

*agustina.c.cottet@gmail.com

Citar este artículo

COTTET, A. C. \& M. I. MESSUTI. 2019. Dos nuevos registros de musgos (Bryophyta) para la Argentina. Bol. Soc. Argent. Bot. 54: 487-491.

DOI: http://dx.doi. org/10.31055/1851.2372.v54. $\mathrm{n} 4.24114$

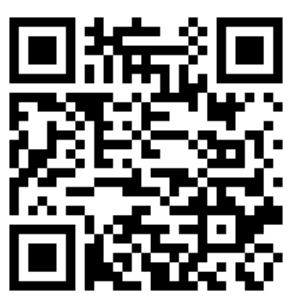

Recibido: 29 Abril 2019 Aceptado: 10 Octubre 2019 Publicado: 15 Diciembre 2019 Editor: Guillermo Súarez (D)

ISSN versión impresa 0373-580X ISSN versión on-line 1851-2372

\section{SUMMARY}

Background and aims: Although the flora of mosses in Argentina has been extensively studied, there are still areas to explore and taxa to register. The aim of this study was to record Fabronia macroblepharis Schwägr. and Rhynchostegium serrulatum (Hedw.) A. Jaeger for Argentina in the National Park Lago Puelo, Chubut.

M\&M: The material examined was studied according to the traditional techniques for bryophytes.

Results \& Conclusions: In this contribution Fabronia macroblepharis and Rhynchostegium serrulatum are cited for the first time for Argentina. Descriptions, illustrations, distribution and comments of each of them are here presented.

\section{KEYWORDS}

Argentina, Bryophyta, Fabronia, Rhynchostegium.

\section{RESUMEN}

Introducción y objetivo: Si bien la flora de musgos de la Argentina ha sido ampliamente estudiada aún quedan ambientes por explorar y taxones para registrar. El objetivo de este trabajo fue registrar Fabronia macroblepharis Schwägr. y Rhynchostegium serrulatum (Hedw.) A. Jaeger para la Argentina en el Parque Nacional Lago Puelo, Chubut.

M\&M: El material examinado fue estudiado de acuerdo a las técnicas tradicionales para las briofitas.

Resultado y Conclusión: En esta contribución se citan por primera vez a Fabronia macroblepharis y Rhynchostegium serrulatum para la Argentina. Se presentan descripciones, ilustraciones, distribución y comentarios para cada una de ellas.

Palabras clave

Argentina, Bryophyta, Fabronia, Rhynchostegium.

\section{INTRODUCCIÓN}

Si bien la flora de musgos de la Argentina ha sido ampliamente estudiada [por ejemplo: Müller (1881, 1897), Schiavone (1988, 1993), Schiavone \& Sarmiento (1985), Schiavone \& Biasuso (1997), Suárez \& Schiavone (2010) y Jimenez et al., (2015) para la región norte; Hosseus (1937, 1938, 1939, 1940), para el centro y noreste, y Dusén $(1903,1906)$, Cardot (1905, 1908), Cardot \& Brotherus (1923) y Kühnemann (1938, 1944) Matteri (1968, 1972, 1980, 1982, 1983, 1984, 1986, 1989), Kühneman \& Gonçalves-Carralves (1975, 1979), Fife \& Matteri (1984), Rodríguez de Sarmiento \& Schiavone $(1988,1993)$, Matteri \& Ochira (1989), Matteri \& Schiavone (1988, 1991), Matteri \& Hyvönen (1992) Rovere \& Calabrese (2011) y Cottet et al., (2016) para la región austral] (fide Matteri, 2003; Suárez \& Schiavone, 2010; Rovere \& Calabrese, 2011; Jimenez et al., 2015; Cottet et al., 2016) aún quedan ambientes inexplorados con taxones por registrar. 
En este trabajo se cita por primera vez para la Argentina a Fabronia macroblepharis Schwägr. (Fabroniaceae) and Rhynchostegium serrulatum (Hedw.) A. Jaeger (Brachytheciaceae). Estas especies fueron encontradas creciendo en bosques dominados por Nothofagus spp. en el noroeste de la provincia de Chubut, Argentina. Se incluyen descripciones, ilustraciones, distribución y comentarios de cada una de las especies.

\section{Materiales y Métodos}

El estudio se basa en ejemplares coleccionados en el Parque Nacional Lago Puelo, provincia de Chubut, Argentina, depositados en el herbario BCRU y en la revisión bibliográfica que incluye las especies estudiadas tales como Buck (1983, 1994), McFarland (1994), Calabrese (1995), Buck (1998) y Fuertes \& Marchessi (2013). El material examinado fue procesado de acuerdo a las técnicas convencionales para el estudio de las briofitas (Frahm, 2003).

\section{Resultados}

\section{Tratamiento taxonómico}

Fabronia macroblepharis Schwägr., Spec. Musc. Suppl. 3(1): 247a. 1828. TIPO:

BRAZIL, s. loc., on rotting wood, D. Pohl (Holotipo, G n.v.) (Buck, 1983) Fig. 1 A-E.

Plantas verde claro-lustrosas a grisáceas; matas laxas y delicadas de hábito extendido corto ascendente. Tallo rastrero, 1-3 cm, irregularmente ramificado, ramas $0,4-0,7 \mathrm{~cm}$ de longitud; en sección transversal cordón central ausente; rizoides agrupados debajo del tallo; parafilias ausentes; pseudoparafilias foliosas; pelos axilares de dos células, la basal corta y la distal alargada. Hojas del tallo y las ramas indiferenciadas, las de las ramas levemente menores. Hojas de las ramas erectas cuando secas, extendidas cuando húmedas, ovado a ovado-lanceoladas, 0,4-0,6 × 0,2-0,5 mm, ápice largo-acuminado; margen plano, entero en la base, dentado hasta ciliado hacia el ápice; costa $3 / 4$ del largo de la lámina, débil; células de la lámina lisas, paredes delgadas, células apicales y superiores oblongo-fusiformes hasta lineares; células medias largo-hexagonales, 50-65 × 9-13 $\mu \mathrm{m}$; región alar diferenciada, cuadradas, 20-32 × 13-17 $\mu \mathrm{m}$. Hojas periqueciales ovado-lanceoladas, $0,6-0,8 \times 0,4-$ $0,5 \mathrm{~mm}$. Seta elongada, $2-4 \mathrm{~mm}$ de longitud, erecta o levemente curvada, lisa. Cápsula erecta, urna ovoide, $0,1-0,15 \times 0,05-0,07 \mathrm{~mm}$, células del exotecio cuadradas hasta corto-rectangulares; estomas faneróporos en el cuello de la cápsula. Peristoma simple, 16 dientes en 8 pares, 250$200 \mu \mathrm{m}$ de longitud, papilosos. Opérculo cónico. Caliptra lisa. Esporas ovoides, 30-35 × 18-22 $\mu \mathrm{m}$, fuertemente papilosas.

Distribución. Esta especie se distribuye desde México hasta el sur de Brasil (Buck, 1998). En este trabajo se cita por primera vez para la Argentina (Fig. 2).

Comentarios. Esta especie se caracteriza por ser de color gris verdoso, tener hojas apresadas, oblongo-lanceoladas y gradualmente acuminadas con el margen dentado que se extiende muy por debajo de la lámina media (Buck, 1998). La mayoría de las características observadas en el material examinado coinciden con la descripción presentada por Buck (1998). Sin embargo, se observan diferencias en la forma y en el tamaño de las esporas (ovoides, 30-35 $\times 18-22 \mu \mathrm{m}$ ), respecto a la descripción presentada por Buck (1998) (globosas, 10-17 $\mu \mathrm{m}$ ).

Fabronia ciliaris (Brid.) Brid., también presente en los bosques andinos patagónicos, se diferencia de F. macroblepharis por presentar hojas ovadas a lanceoladas extendidas y por sus hojas periqueciales más pequeñas que las rameales (Buck, 1994, 1998).

Material examinado. ARGENTINA. Chubut: Depto. Cushamen, Parque Nacional Lago Puelo, Sendero Jardín Botánico, sobre suelo, 19/I/2014, A. C. Cottet (BCRU 5436); ibid., Sendero La Voz del Arce, sobre suelo, 01/II/2014, A. C. Cottet (BCRU 5439); ibid., 30/VIII/2014, A. C. Cottet (BCRU 5449).

Rhynchostegium serrulatum (Hedw.) A. Jaeger, Ber. Thätigk. St. Gallischen Naturwiss. Ges. 187677: 370. 1878. Hypnum serrulatum Hedw. Spec. Musc. Frond. 238-240, pl. 60. 1801. Eurhynchium serrulatum (Hedw.) Kindb., Ottawa Nat. 7: 21. 1870. (Ignatov, 2014). Brachythecium serrulatum (Hedw.) H. Rob., Bryologist 65: 125. 1962. (Ignatov, 2014). 

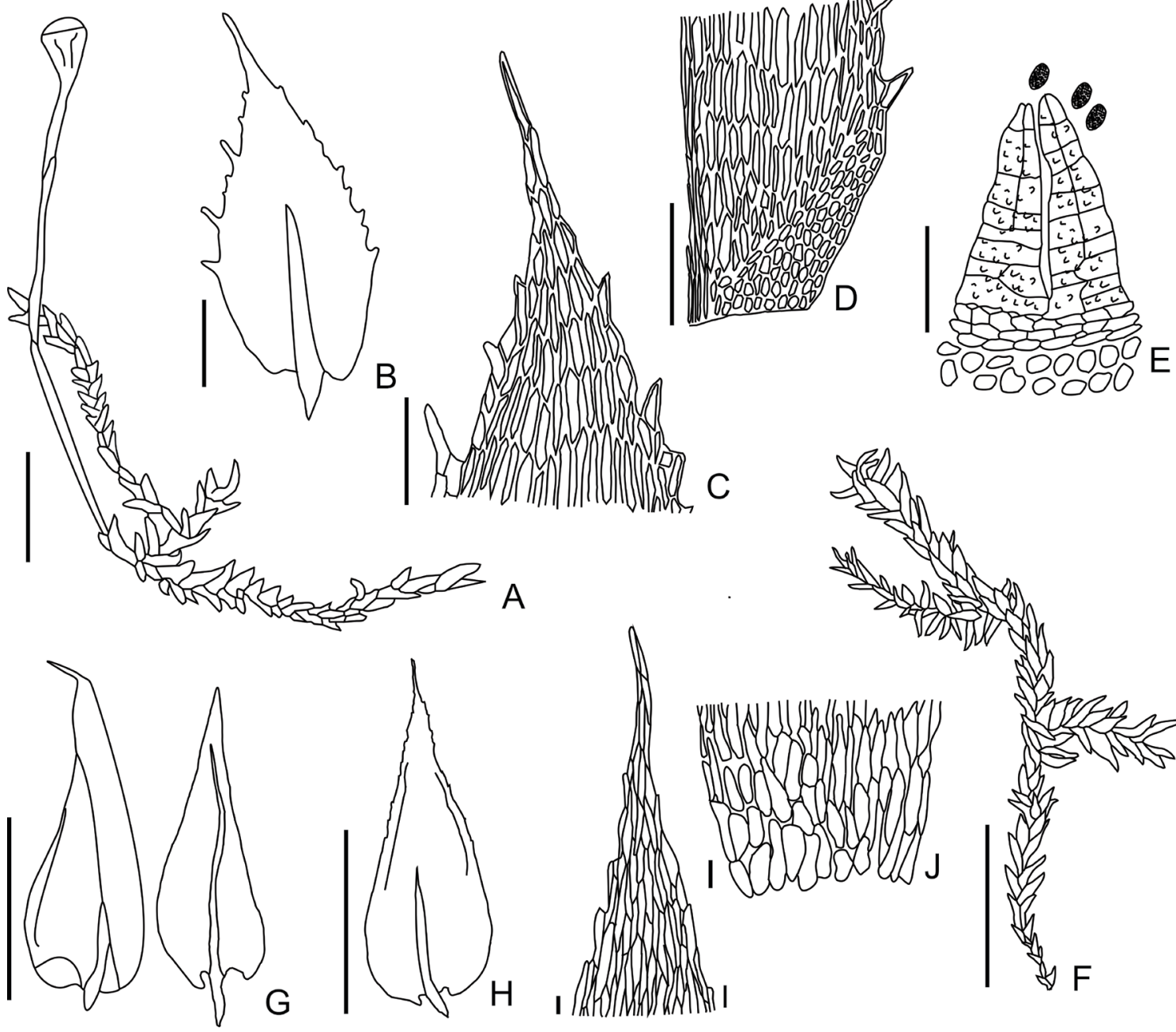

Fig. 1. Fabronia macroblepharis Schwägr. (BCRU 5449) A: Hábito. B: Hojas de la rama C. Detalle del ápice de la hoja de la rama. D: Detalle de la región alar de la hoja de la rama. E: Porción del peristoma con esporas. Rhynchostegium serrulatum (Hedw.) A. Jaeger (BCRU 5455) F: Hábito. G: Hoja del tallo. H: Hoja de la rama. I: Detalle del ápice de la hoja de la rama. J: Detalle de la región alar de la hoja de la rama. Escala= F: $1 \mathrm{~cm} ; \mathrm{A}, \mathrm{G}, \mathrm{H}: 1 \mathrm{~mm}$; B, C, D, E: $100 \mu \mathrm{m}$; I, J: $10 \mu \mathrm{m}$.

Steerecleus serrulatus (Hedw.) H. Rob., Mem. New York Bot. Gard. 45: 678-681. 1987. (Ignatov, 2014). TIPO: Ind. loc. «Lancastrina in Pensylvania lecta specimina terrestris» (Tipo no localizado) (Fuertes \& Marchessi, 2013). Fig. 1 F-J.

Plantas verde oscuras, amarillentas o doradas, brillantes; matas más o menos laxas a frondosas. Tallos extendidos, 4-7 cm de longitud; en sección transversal 2-3 hileras de células pequeñas con paredes engrosadas rodeando células grandes de paredes delgadas, cordón central presente; pseudoparafilias foliosas. Hojas de los tallos y ramas muy similares, erecto-extendidas a complanadas en estado seco y húmedo, ovado-lanceoladas, 1,4-2,3 × 0,4-0,8 $\mathrm{mm}$, lisas a levemente plicadas distalmente, ápice largo acuminado, algo retorcido, base levemente decurrente; margen plano, serrulado hacia el ápice; costa débil, 1/2-3/4 de la longitud de la lámina; células apicales similares a las medias, lineares, con paredes 


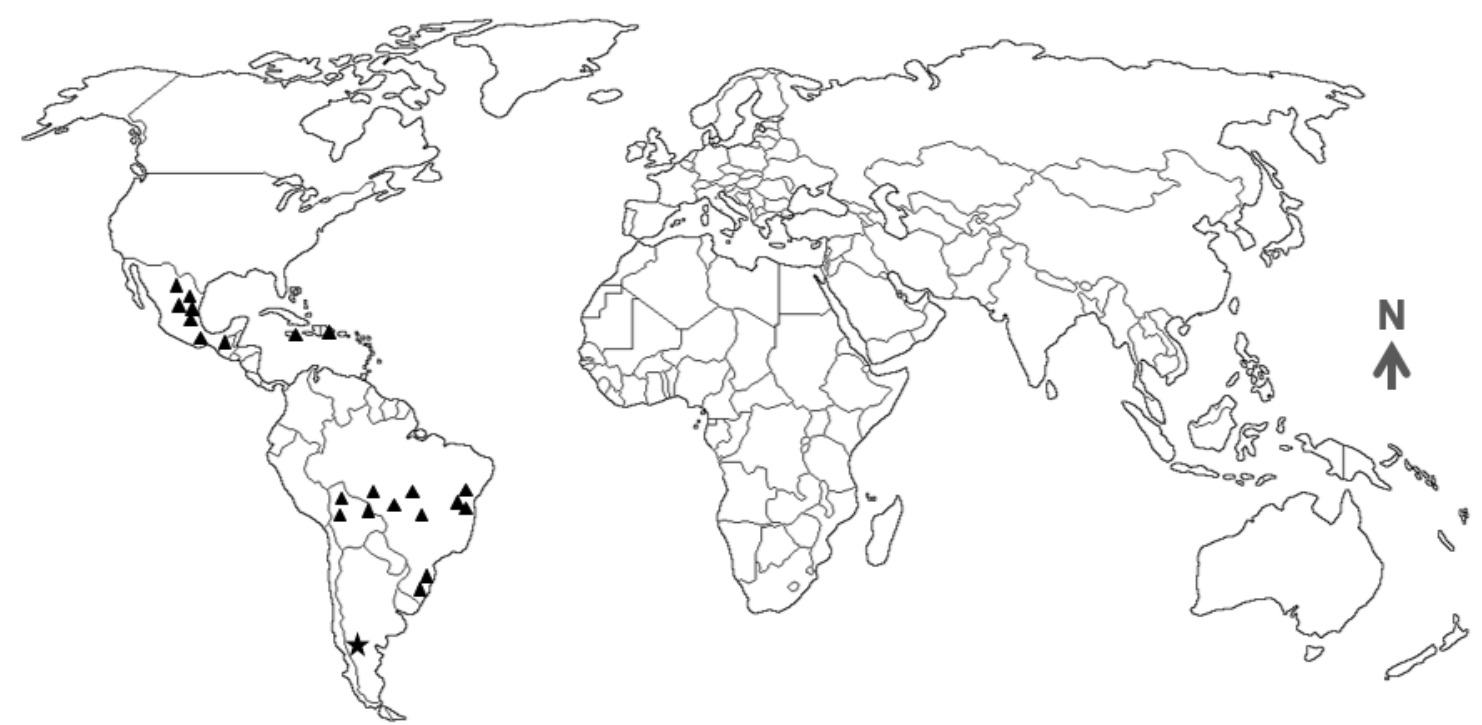

Fig. 2. Mapa de distribución global de Fabronia macroblepharis Schwägr. Registros previos (triángulos), nuevo registro (estrella).

poco engrosadas, $52-80 \times 6,5-9,4 \mu \mathrm{m}$; región alar poco desarrollada, con células cuadradas a cortorectangulares. Autoicas. Hojas periqueciales largoacuminadas. Seta elongada, 1-1,5 cm de longitud, lisa, levemente retorcida. Cápsula inclinada, urna ovoide a cilíndrica, 1-1,5 × 0,7-1 mm; células exoteciales corto rectangulares; dientes del exostoma marrones, triangulares, basalmente estriados y distalmente papilosos, 550-560 $\mu \mathrm{m}$ de longitud; endostoma papiloso, membrana basal alta, cilias presentes generalmente en grupos. Esporas esféricas, 15-17 $\mu \mathrm{m}$, mayormente lisas.

Distribución. Esta especie se distribuye en el Este de América del Norte, América Central y el norte de los Andes de América del Sur y Uruguay, Japón y Corea (McFarland, 1994; Buck, 1998; Fuertes \& Marchessi, 2013). Se la registra por primera vez para Argentina (Fig. 3).

Comentarios. Las especies incluidas en el género Rhynchostegium pueden ser confundidas con las del género Eurhynchum Bruch \& Schimp. Este último también incluido en la familia Brachytheciaceae. Sin embargo, Rhynchostegium serrulatum tiene las hojas del tallo y de las ramas similares (ovado-lanceoladas); mientras que Eurhynchum fuegianum Card., también presente en los bosques andino patagónicos dominados por Nothofagus spp., posee diferenciación entre las hojas de tallo y de las ramas (ovado-cordadas y ovadas respectivamente) (Calabrese, 1995).

Material examinado. ARGENTINA. Chubut: Depto. Cushamen, Parque Nacional Lago Puelo, Sendero Jardín Botánico, sobre suelo, 21/II/2014, A. C. Cottet (BCRU 5434); ibid., Sendero Pitranto Grande, sobre suelo, 24/XI/2014, A. C. Cottet (BCRU 5455).

\section{Contribución DE LOS AUtORES}

ACC coleccionó el material examinado. Ambas autoras realizaron la investigación y preparación del manuscrito.

\section{Agradecimientos}

Las autoras agradecen a A. W. Archer (NSW, Australia) y a G. M. Suárez (LIL, Argentina) por sus comentarios sobre la preparación de este el manuscrito. Este trabajo fue financiado por el CONICET y la UNComahue. 


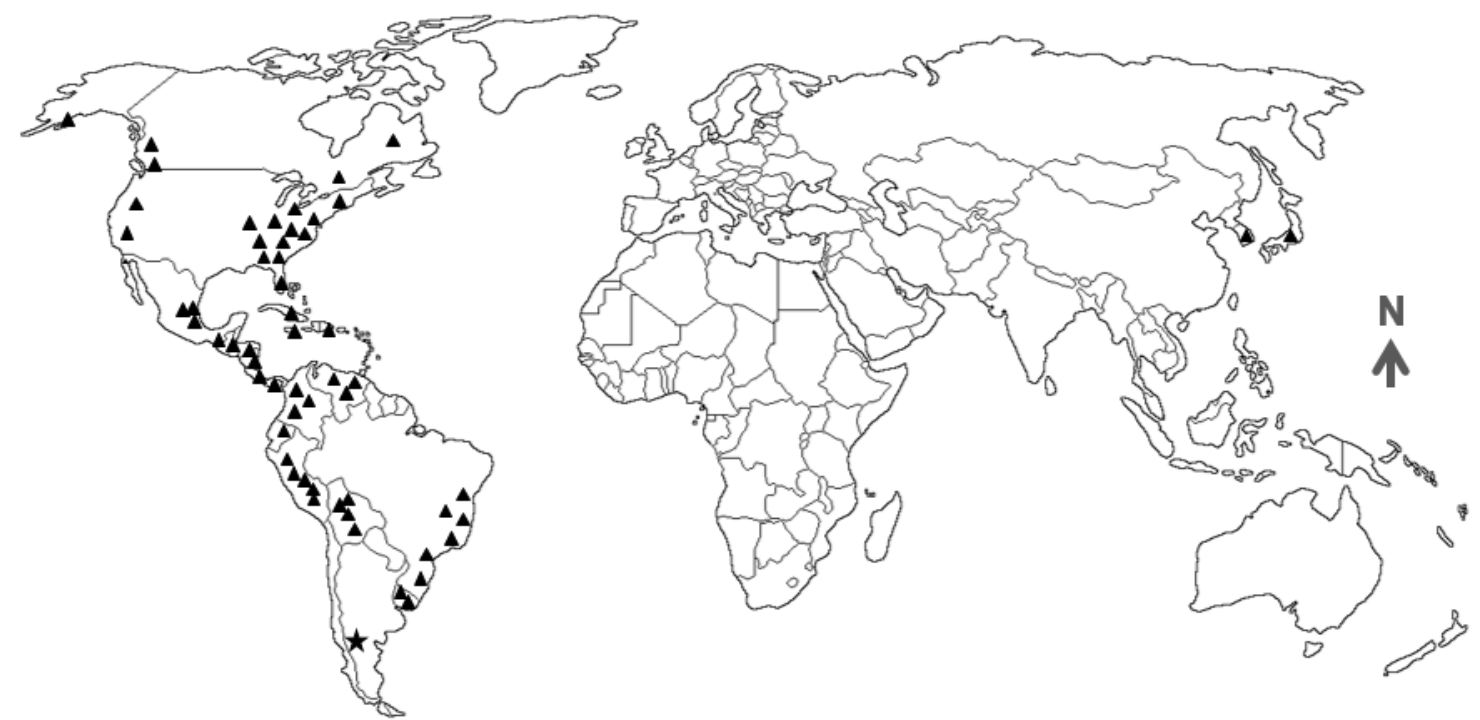

Fig. 3. Mapa de distribución global de Rhynchostegium serrulatum (Hedw.) A. Jaeger. Registros previos (triángulos), nuevo registro (estrella).

\section{Bibliografía}

BUCK, W. R. 1983. A Synopsis of the South American Taxa of Fabronia (Fabroniaceae). Brittonia 35: 248-254.

BUCK, W. R. 1994. Fabroniaceae. En: SHARP, A. J., H. CRUM \& P. M. ECKEL (eds.), The Moss Flora of Mexico. Mem. New York Bot. Gard. 69: 860-867.

BUCK, W. R. 1998. Pleurocarpous mosses of the West Indies. Mem. N. Y. Bot. Gard. 82: 1-400.

CALABRESE, G. M. 1995. Flora muscinal de Puerto Blest y alrededores (Parque Nacional Nahuel Huapi, Argentina). Trabajo para optar al grado de Licenciado en Ciencias Biológicas. Centro Regional Universitario Bariloche. Universidad Nacional del Comahue. San Carlos de Bariloche.

COTTET, A. C., G. M. SUÁREZ, M. M. SCHIAVONE \& M. I. MESSUTI. 2016. Cryphaea consimilis (Cryphaeaceae, Bryophyta), nueva cita para la Argentina. Bol. Soc. Argent. Bot. 51: 219-222.

FRAHM, J. P. 2003. Manual of tropical bryology. Trop. Bryol. 23: $1-200$.

FUERTES, E. \& J.E. MARCHESSI. 2013. Notula taxonómica, nomenclatural y corológica de Steerecleus (Bryophyta: Brachyteciaceae) en Uruguay. Bot. Complut. 37: 35-39. http://dx.doi.org/10.5209/rev_BOCM.2013.v37.42266
IGNATOV, M. S. 2014. Brachytheciaceae. En: Flora of North America Editorial Committee (eds.). Flora of North America North of Mexico, Vol. 28, pp. 404-468. Oxford University Press, New York and Oxford.

JIMENEZ M. S., M. M. SCHIAVONE, G. M. SUÁREZ, \& C. DELGADILLO. 2015. Neosharpiella aztecorum H. Rob. \& Delgad. (Gigaspermaceae), new to the bryophyte flora of South America. Cryptogam. Bryol. 36: 69-74.

MATTERI, C. M. 2003. Los Musgos (Bryophyta) de la Argentina. Trop. Bryol. 24: 33-100

MCFARLAND, K. D. 1994. Rhynchostegium. En: SHARP, A. J., H. CRUM \& P. M. ECKEL (eds.), The Moss Flora of Mexico. Mem. New York Bot. Gard.69: 932-941.

ROVERE, A. E. \& G. M. CALABRESE. 2011. Diversidad de musgos en ambientes degradados sujetos a restauración en el Parque Nacional Lago Puelo (Chubut, Argentina). Rev. Chil. Hist. Nat. 84: 571-580.

SUÁREZ, G. M. \& M. M. SCHIAVONE. 2010. La familia Cryphaeaceae (Bryophyta) en los bosques del noroeste de Argentina. Bol. Soc. Argent. Bot. 45: 29-45. 
